\title{
Application of Uniform Experimental Design in Optimizing Excitation Parameters for Magnetic Frequency Mixing Measurements
}

\author{
Yu Chang ${ }^{1}$, Jingpin Jiao ${ }^{1 *}$, Xiucheng Liu' ${ }^{1}$ Guanghai $\mathrm{Li}^{2}$, Cunfu $\mathrm{He}^{1}$ and Bin Wu${ }^{1}$
}

\begin{abstract}
Excitation parameter preferences are key factors affecting the performance of magnetic frequency mixing detection. A uniform experimental design method was used to analyze this influence. Using fuzzy theory, a comprehensive model is established for evaluating the effect of magnetic frequency mixing. A polynomial is selected as the regression function to express explicitly the correlation between the excitation parameters and the frequency-mixing effect. The excitation parameters were then optimized using genetic algorithm. Magnetic frequency mixing experiments were conducted to measure the surface hardness of some ferromagnetic materials. Frequency mixing is further enhanced under the optimal settings, resulting in an improvement in the measurement sensitivity. The results of this study support the application of the magnetic frequency mixing technique in non-destructive testing.
\end{abstract}

Keywords: Magnetic frequency mixing technology, Excitation parameters, Uniform experimental design, Regression statistical method, Surface hardness measurement

\section{Introduction}

The frequency-mixing technique is an effective means for the extraction of weak nonlinear signals from noisy backgrounds $[1,2]$, and widely used in many fields, such as spectroscopy, ultrasonics, and electronics [3, 4]. The magnetic frequency mixing technology is of particular interest here $[5,6]$. In experiments employing magnetic frequency mixing, a ferromagnetic material is usually exposed to an AC magnetic field consisting of two frequencies [7]. The low-frequency component of the magnetic field drives periodically the material into its saturation magnetization regime, whereas the high-frequency component is weaker and can only be dispersed near the surface of the material. Affected by the two magnetic fields, the ferromagnetic material undergoes magnetic domain rotation and domain wall motion, which results in frequency mixing [8]. Because

\footnotetext{
*Correspondence: jiaojp@bjut.edu.cn

${ }^{1}$ College of Mechanical Engineering and Applied Electronics Technology,

Beijing University of Technology, Beijing 100124, China

Full list of author information is available at the end of the article
}

frequency-mixing is related to the hysteretic nonlinearity of ferromagnetic materials $[9,10]$, the magnetic frequency mixing technique is sensitive to the microstructure evolution of ferromagnetic materials. Compared with traditional magnetic techniques, it has a higher sensitivity and better signal-to-noise ratio [11].

As frequency-mixing is generated in the nonlinear interaction between two magnetic fields and the magnetic microstructure, the excitation parameters for the two magnetic fields inevitably influence the performance of magnetic frequency mixing detection given the same test ferromagnetic material [12-14]. Burdin et al. [15] analyzed the influence of the frequency and amplitude of the high-frequency magnetic field on frequency mixing effect by experiment. The optimal excitation frequency for the high-frequency magnetic field was determined from frequency sweeping experiments. Rabehi et al. [16] studied the effect of the current intensity in the excitation coil on the magnetic field theoretically and experimentally. A planar micro-coil was designed subject to optimal coil-dimension constraints. However, previous research on excitation parameter preferences was limited 
to the effect of a single factor. The supposition was that if only one factor was varied, the preference is certainly the influence of that single variable. Clearly, this method does not reveal the combined effects of multiple factors that may be coupled [17].

Common statistical experimental design methods for multi-factor optimization include orthogonal experimental design (OED) $[18,19]$ and uniform experimental design (UED) $[20,21]$. OED is a multi-factor experimental design method based on the orthogonal array. It selects representative points from the full factorial experiment that are distributed uniformly within the test range [22]. The OED method has been used in parameter optimizations in magnetic measurements. For example, Wu et al. [23] applied the OED method to multi-parameters optimization in magnetic flux leakage testing. The parameters include thickness, height and shielding layer of the excitation coil. Experimental results showed that the sensitivity of detection in steel cables was greatly improved using the OED method, and thereby reduces the number of required experiments and achieves significant results. However, the OED method only obtains the optimal values of representative sets of experiments, but not the optimum values over all experimental ranges. In addition, it is no longer valid when the experimental level is comparatively high.

The UED is similarly a multi-factor experimental design method but based on the quasi-Monte Carlo method of number theory [24]. The principle of UED is to replace the complete combination of all possible experimental parameters by a small number of experimental trials uniformly distributed within the parameter space [25]. The parameter values for the trials are determined using number theoretic method; therefore, the chosen trials prove that the values approximate well the complete combination of experimental parameters. Compared with conventional statistical experimental design methods, such as the Latin square design and the OED method, the UED method accommodates the largest possible number of levels for each excitation parameter among all experimental designs [24-27]. In practice, the UED method has wide applications in engineering $[28,29]$.

The objective of this study is to improve the performance of magnetic frequency mixing detection using excitation parameter optimization. The influence of the amplitudes and frequencies of high-frequency and lowfrequency magnetic fields on the performance of magnetic frequency mixing testing was analyzed using UED. Specifically, a comprehensive model was established for evaluating the effect of magnetic frequency mixing. The regression method from the field of statistics has been used to analyze the variation of excitation parameters versus frequency-mixing effect. The excitation parameters were then optimized using genetic algorithm.

\section{Parameters Optimization Based on Uniform Experimental Design}

Generally, the magnetic frequency mixing effect is a kind of weak signal. The higher the strength of the pickup signal, the better the detection of the mechanical properties of the material. The intensity of magnetic frequency mixing effect is affected by the excitation condition. The relationship between them can be described by Figure 1 . In general, there are two forms of magnetic frequency mixing effect, which are reflected as the mixing components in the spectrum and the minor hysteresis loops in the hysteresis loop. Based on the simulation, the strength of the mixing component is positively correlated with the area of the minor hysteresis loop (Figure 2). And the local hysteresis loss is proportional to the area of the minor hysteresis loop according to the hysteresis theory. Therefore, the influence of excitation conditions on magnetic frequency mixing effect can be reflected by its relationship with minor hysteresis loss.

Generally, the total minor hysteresis loss is expressed as the sum of hysteresis loss $W_{\mathrm{h}, \mathrm{m}}$, excess loss $W_{\text {exc, } \mathrm{m}}$ and eddy current loss $W_{\mathrm{cl}, \mathrm{m}}$. The frequency-dependent dynamic loss $W_{\text {exc,m }}$ and $W_{\mathrm{cl}, \mathrm{m}}$ terms can be expression by the excitation frequency ratio and amplitude ratio. It can be written as

$$
\begin{aligned}
& W_{\mathrm{exc}, \mathrm{m}}=2 \sum_{i=1}^{m_{f} / 2} 8 \sqrt{\sigma G S V_{0} J_{m, i}} J_{m, i}^{3 / 2} \sqrt{g_{m, i}\left(m_{a}, m_{f}\right)} f_{L}^{1 / 2}, \\
& W_{\mathrm{cl}}=\frac{4}{3} \sigma d^{2} J_{p}^{2} g_{M}\left(m_{a}, m_{f}\right)^{2} f_{L},
\end{aligned}
$$

where $\sigma$ is the conductivity, $d$ is the thickness of specimen, $G=0.1356$ is a dimensionless coefficient, $S$ is the cross-sectional area, $V_{0}$ is a parameter defining the statistics of the magnetic objects, $J_{p}$ is the sum of amplitudes of high and low excitation magnetic field, $m_{a}$ is the ratio of

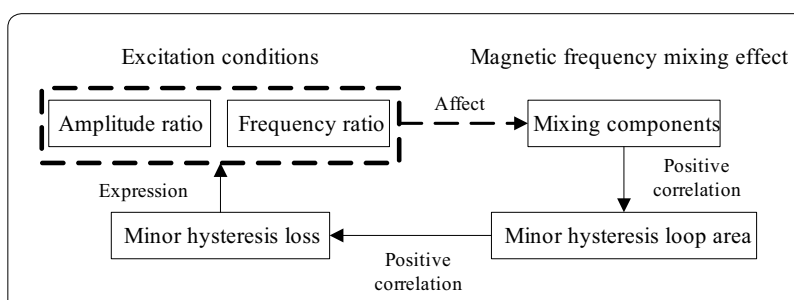

Figure 1 Schematic of the relationship between magnetic frequency mixing excitation conditions and frequency mixing effect 


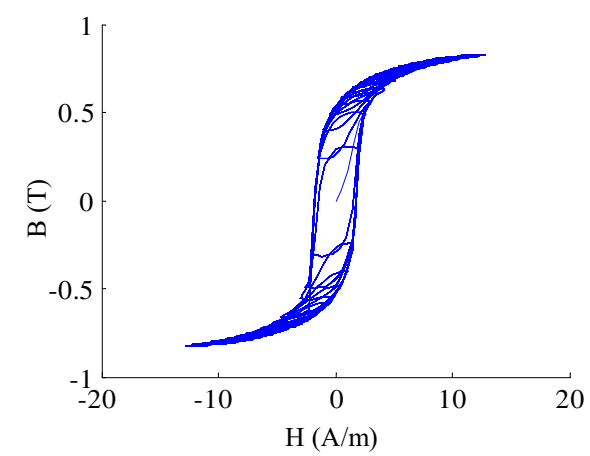

a Area of the minor hysteresis loop is big

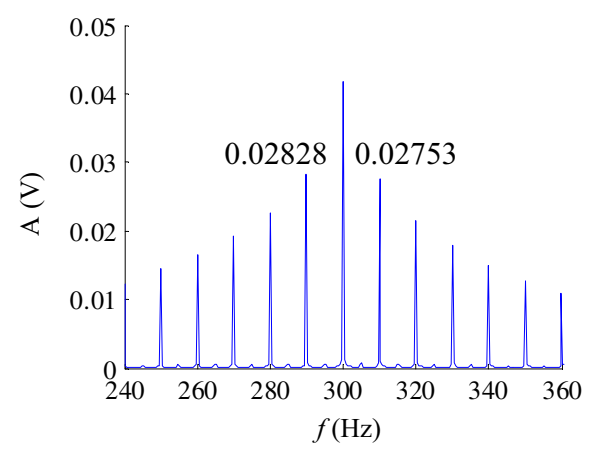

b Strength of the mixing component is high

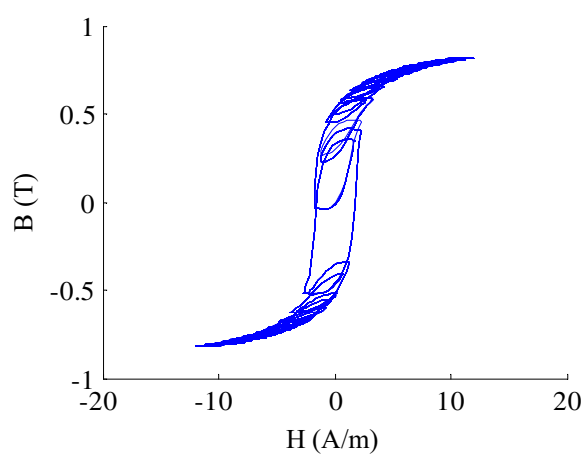

c Area of the minor hysteresis loop is small

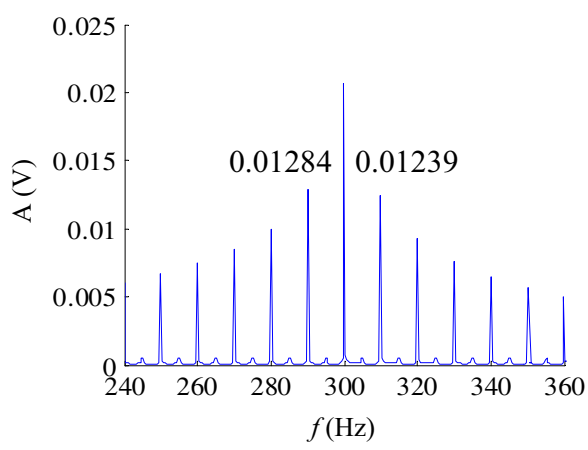

d Strength of the mixing component is low

Figure 2 Relationship between the area of minor hysteresis loop and the strength of mixing component high and low frequency amplitudes, $m_{f}$ is the ratio of high and low frequency. $J_{m, i}, g_{m, i}$ and $g_{M}$ written as

$$
\begin{aligned}
& J_{m, i}=J_{p} \frac{g_{M}\left(m_{a}, m_{f}\right)}{g_{m, i}\left(m_{a}, m_{f}\right)}, \\
& g_{m, i}\left(m_{a}, m_{f}\right) \cong \frac{m_{f}}{1-m_{a} \times \sin \left[2 \pi(3 / 4+i-1) / m_{f}\right]}, \\
& g_{M}\left(m_{a}, m_{f}\right) \cong \frac{m_{f}}{1+2 m_{a} \times \sum_{i=1}^{m_{f} / 2} \sin \left[2 \pi(3 / 4+i-1) / m_{f}\right]} .
\end{aligned}
$$

The typical results of frequency-dependent minor hysteresis loss predicted by Eqs. (1) and (2) is shown in Figure 3.

From Figure 3 we can see that the frequency-dependent minor hysteresis loss varies with the excitation amplitude ratio and the frequency ratio. If the amplitude ratio or frequency ratio is constant, the result is just a conditional extremum of the dynamic loss. The best extremum among the feasible region of four excitation parameters cannot be obtained. Therefore, it can be inferred from Figures 1, 2 and 3 that the magnetic frequency mixing effect is jointly affected by four excitation parameters. The optimization of the combination of excitation parameters is beneficial to improve the magnetic frequency mixing effect.

Figure 4 shows a flow diagram of the parameter optimization of magnetic frequency mixing testing using the UED method. Considering the four influencing factors, amplitudes and frequencies of the low-frequency and high-frequency components of the magnetic field, the magnetic frequency mixing experiments were arranged in accordance with the UED method. Two characteristic indexes were extracted from the measured signals, and a comprehensive model was established for evaluating the effect of magnetic frequency mixing. A multivariate equation was developed by regression analyzing the four excitation parameters, which were then optimized using the genetic algorithm.

\subsection{Experimental System of Magnetic Frequency Mixing}

An experimental verification of the optimization of the excitation parameters was obtained from magnetic frequency mixing tests of hardness of several ferromagnetic materials (Figure 5). The system includes a data acquisition board, power amplifier, magnetic frequency-mixing sensor, specimen and computer. The sensor is composed of a U-shaped magnetic core, excitation coil and pick-up coil. A superimposed electric signal with high-frequency and low-frequency components, is generated by the excitation 


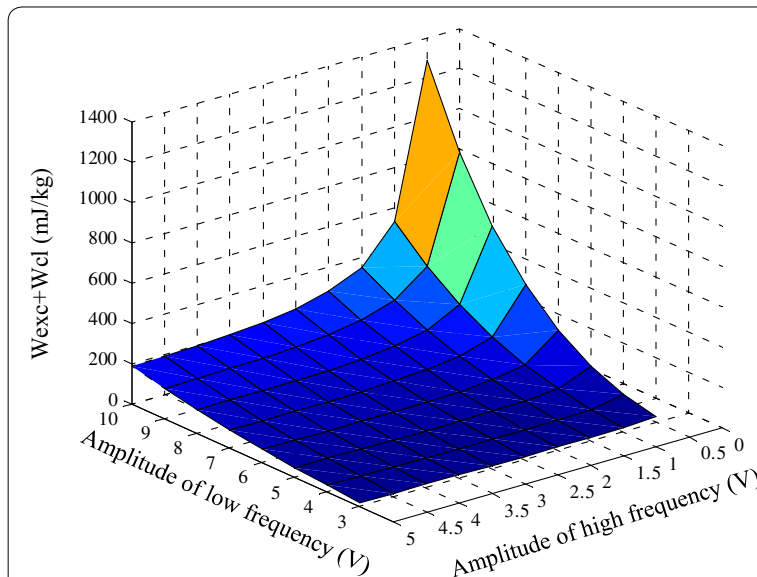

a When the $m_{f}$ is 200 , dynamic loss varies with $m_{a}$

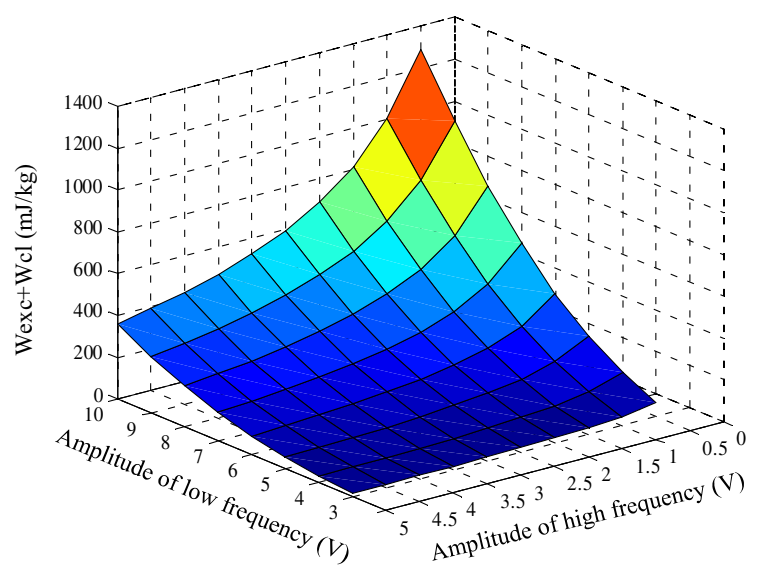

c When the $m_{f}$ is 80 , dynamic loss varies with $m_{a}$

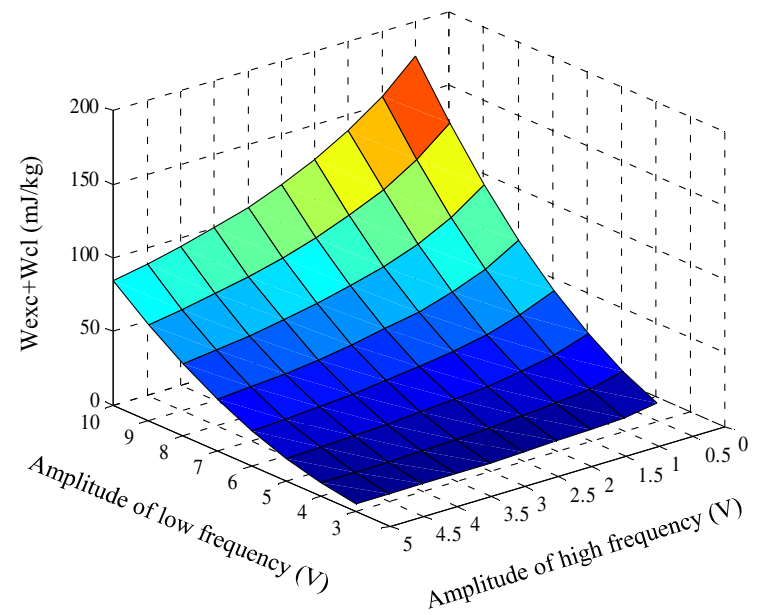

e When the $m_{f}$ is 200 , dynamic loss varies with $m_{a}$

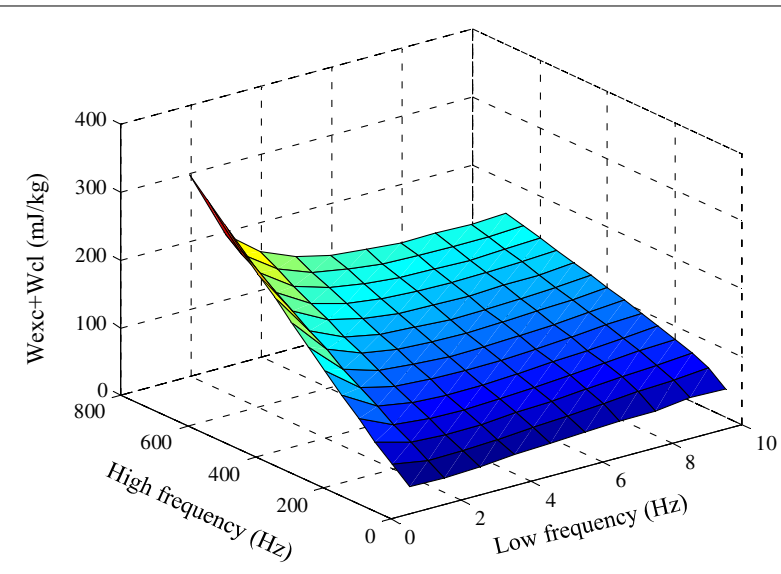

b When the $m_{a}$ is 5 , dynamic loss varies with $m_{f}$

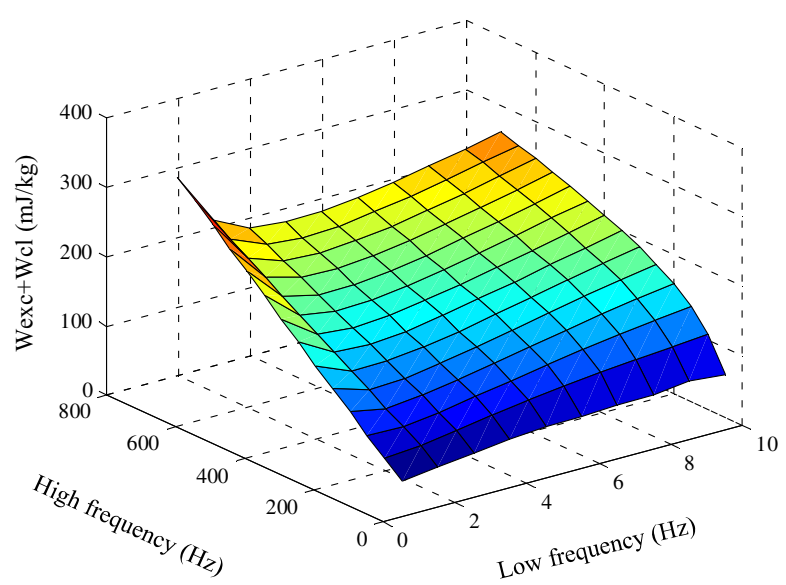

d When the $m_{a}$ is 2.8 , dynamic loss varies with $m_{f}$

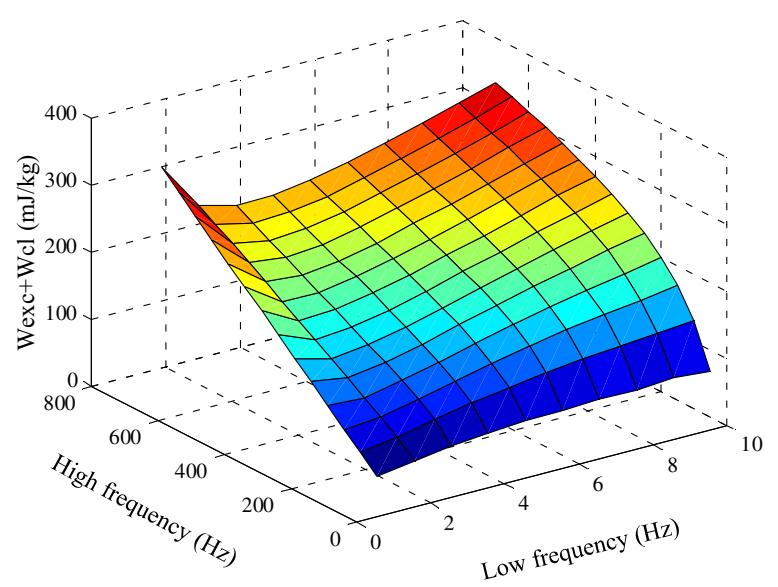

f When the $m_{a}$ is 1.4 , dynamic loss varies with $m_{f}$

Figure 3 Distribution of minor dynamic loss with excitation frequencies and amplitudes 


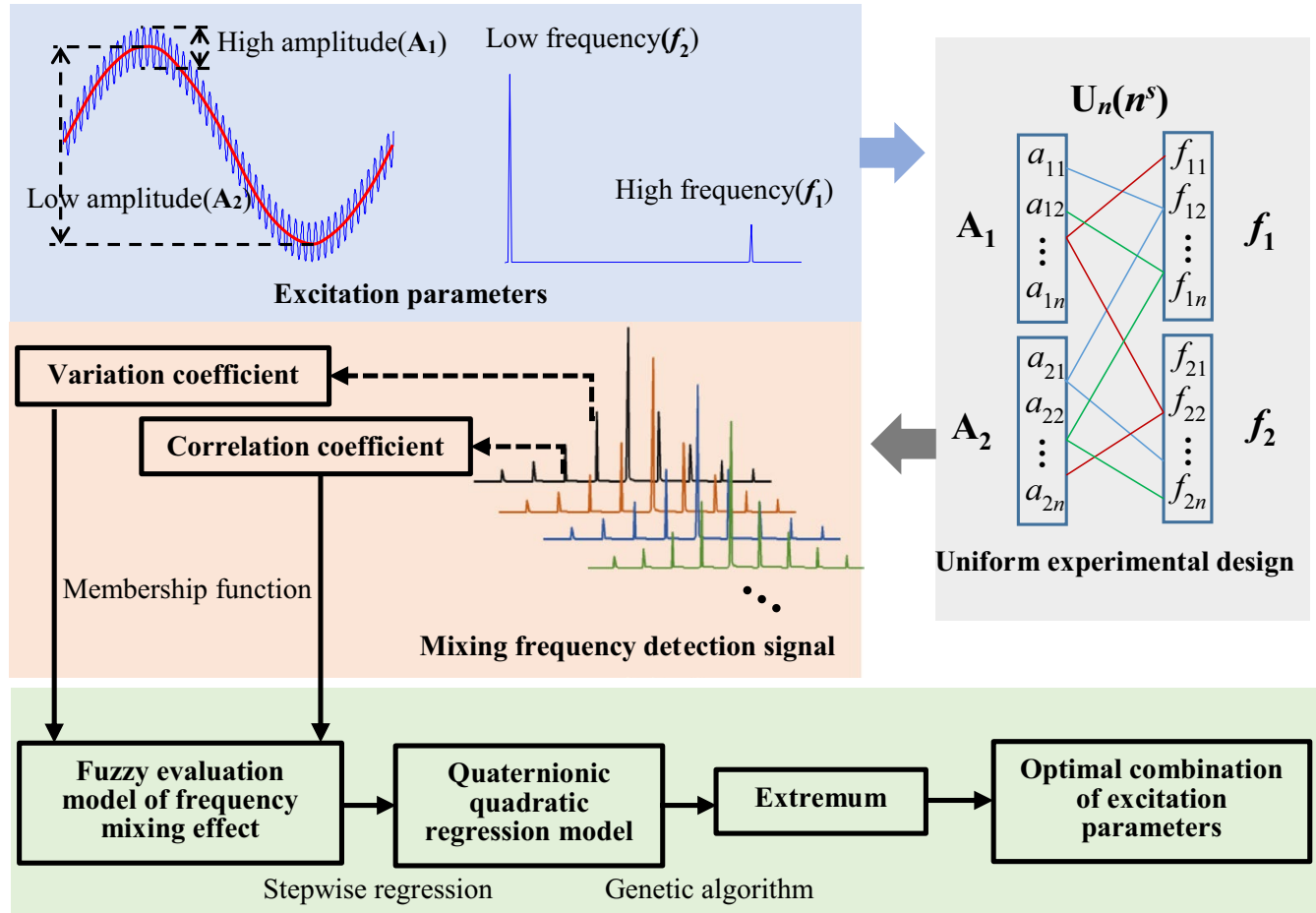

Figure 4 Excitation parameters optimization of magnetic frequency mixing based on uniform experimental design

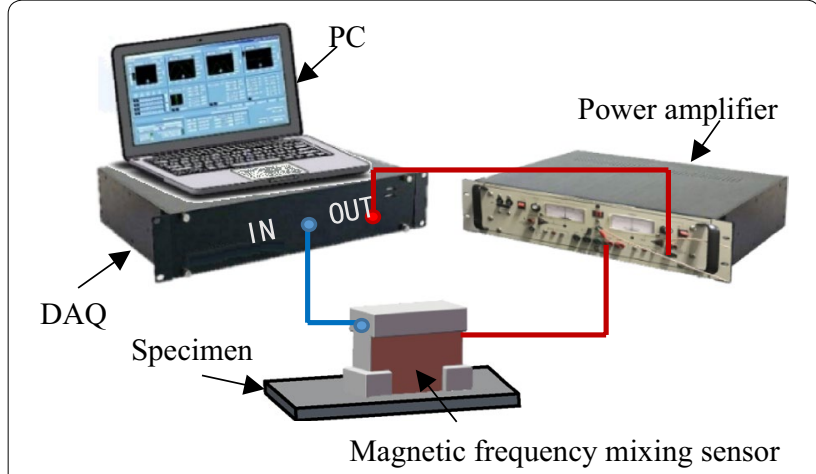

Figure 5 Schematic diagram of the experimental setup for verification

module on the board. The superimposed signal was amplified and then applied to the excitation coil of the sensor. The alternating magnetic field generated by the coil forms a magnetic circuit between the magnetic core and specimen. The pick-up coil induces an alternating electric field in the magnetic circuit. The data sampling card samples the output of the pick-up coil that is subsequently processed by computer.

Magnetic frequency mixing experiments were conducted on nine medium carbon steel specimens. Each specimen was of identical dimensions, $100 \mathrm{~mm} \times 100 \mathrm{~mm} \times 6 \mathrm{~mm}$. The specimens were quenched at $1030{ }^{\circ} \mathrm{C}$ and then tempered at different temperatures to achieve different surface hardenings. The hardness of each specimen was measured by a Vickers indenter. Tables 1 and 2 show the essential component and the Vickers hardness of each specimen.

From Table 2, the surface hardness of all specimens is within a range from 194.0 HV to 595.0 HV. Moreover, with increasing tempering temperature, surface hardness decreases.

\subsection{Uniform Experimental Design}

The frequency and amplitude of both high and low-frequency components of the magnetic field show an effect on the performance of magnetic frequency mixing testing. With previous results from such testing, Table 3 lists the reasonable ranges and steps of four excitation parameters; Table 4 presents the UED table $U_{15}\left(15^{4}\right)$ of the excitation

Table 1 Essential component of steel specimen (wt\%)

\begin{tabular}{llllllll}
\hline Material & $\mathbf{C}$ & $\mathrm{Cr}$ & Mn & Ni & P & S & Si \\
\hline LG1500HS & $0.42-0.50$ & $\leq 0.25$ & $0.50-0.80$ & $\leq 0.25$ & $\leq 0.035$ & $\leq 0.035$ & $0.17-0.37$ \\
\hline
\end{tabular}


Table 2 Vickers hardness of steel specimens

\begin{tabular}{|c|c|c|c|c|c|c|c|c|c|}
\hline Tempered temperature $\left({ }^{\circ} \mathrm{C}\right)$ & 850 & 600 & 560 & 530 & 500 & 400 & 330 & 240 & 180 \\
\hline Hardness (HV) & 194.0 & 235.0 & 270.0 & 294.0 & 306.0 & 412.0 & 481.0 & 547.0 & 595.0 \\
\hline
\end{tabular}

Table 3 Reasonable ranges and steps of the excitation parameters

\begin{tabular}{lllll}
\hline Excitation & $\begin{array}{l}\text { Low } \\
\text { frequency } \\
\mathbf{( H z )}\end{array}$ & $\begin{array}{l}\text { High } \\
\text { frequency } \\
\mathbf{( H z )}\end{array}$ & Low amplitude (V) & $\begin{array}{l}\text { High } \\
\text { amplitude } \\
\text { (V) }\end{array}$ \\
\hline Range & $1-29$ & $50-750$ & $0.5-7.5$ & $0.1-2.9$ \\
Step & 2 & 50 & 0.5 & 0.2 \\
\hline
\end{tabular}

Table 4 UED table $U_{15}\left(15^{4}\right)$ for excitation parameter preferences

\begin{tabular}{|c|c|c|c|c|}
\hline \multirow[t]{2}{*}{ Level } & \multicolumn{4}{|l|}{ Factor } \\
\hline & $\begin{array}{l}\text { Low } \\
\text { frequency } \\
(\mathrm{Hz})\end{array}$ & $\begin{array}{l}\text { High } \\
\text { frequency } \\
(\mathrm{Hz})\end{array}$ & $\begin{array}{l}\text { Low } \\
\text { amplitude (V) }\end{array}$ & $\begin{array}{l}\text { High } \\
\text { amplitude } \\
\text { (V) }\end{array}$ \\
\hline 1 & 17 & 700 & 2 & 2.7 \\
\hline 2 & 5 & 600 & 1 & 1.1 \\
\hline 3 & 29 & 450 & 3 & 0.7 \\
\hline 4 & 11 & 750 & 5 & 1.5 \\
\hline 5 & 13 & 350 & 1.5 & 0.1 \\
\hline 6 & 23 & 300 & 4.5 & 2.9 \\
\hline 7 & 27 & 550 & 6 & 2.1 \\
\hline 8 & 7 & 400 & 7 & 2.5 \\
\hline 9 & 19 & 50 & 4 & 0.9 \\
\hline 10 & 1 & 500 & 3.5 & 1.9 \\
\hline 11 & 3 & 250 & 5.5 & 0.5 \\
\hline 12 & 21 & 650 & 6.5 & 0.3 \\
\hline 13 & 15 & 150 & 7.5 & 1.3 \\
\hline 14 & 25 & 200 & 0.5 & 1.7 \\
\hline 15 & 9 & 100 & 2.5 & 2.3 \\
\hline
\end{tabular}

parameter preferences. For each specimen, the magnetic frequency mixing testing was conducted under different conditions (15 levels), and each measurement is repeated three times. Therefore, there are altogether $9 \times 15 \times 3$ sets of signals, which can be used to optimize the excitation parameters.

\subsection{Model for Evaluating the Frequency-Mixing Effect}

To highlight the effect of excitation parameters on the performance of magnetic frequency mixing testing, two coefficients were extracted from the measured signals, and a comprehensive model was established to evaluate the strength of magnetic frequency mixing under different excitations.

\subsubsection{Characteristic Index of Magnetic Frequency Mixing Effect}

The amplitudes of the nonlinear components at lowerorder mixing frequencies are the most widely used parameters in frequency-mixing measurements [2]. The measuring sensitivity is usually affected by the strength and consistency of the nonlinear components. In accordance with statistics theory, two coefficients, a variation coefficient and a correlation coefficient were introduced and used to evaluate the performance of the magnetic frequency mixing testing. The expression for the variation coefficient $D$ is

$$
D=\frac{\sqrt{\frac{1}{N} \sum_{i=1}^{N}\left[\left(A_{i}^{f_{1}-2 f_{2}}+A_{i}^{f_{1}+2 f_{2}}\right)-\frac{1}{N} \sum_{i=1}^{N}\left(A_{i}^{f_{1}-2 f_{2}}+A_{i}^{f_{1}+2 f_{2}}\right)\right]^{2}}}{\frac{1}{N} \sum_{i=1}^{N}\left(A_{i}^{f_{1}-2 f_{2}}+A_{i}^{f_{1}+2 f_{2}}\right)},
$$

where $A_{i}^{f_{1}-2 f_{2}}$ and $A_{i}^{f_{1}+2 f_{2}}$ are the amplitudes of the nonlinear components at $f_{1}-2 f_{2}$ and $f_{1}+2 f_{2}$ in the $i$ th experiment, respectively, and $N$ the number of repeated experiments at all levels ( $N=3$ in this study). The variation coefficient $D$ is a deflection minor-type function meaning that the smaller the value of $D$ is, the higher is the strength of the nonlinear components.

Similarly, the expression for the correlation coefficient $P$ is

$$
P=\frac{\sum_{i=1}^{M}\left(A_{i}^{f_{1}-2 f_{2}}-\bar{A}^{f_{1}-2 f_{2}}\right)\left(A_{i}^{f_{1}+2 f_{2}}-\bar{A}^{f_{1}+2 f_{2}}\right)}{\sqrt{\sum_{i=1}^{M}\left(A_{i}^{f_{1}-2 f_{2}}-\bar{A}^{f_{1}-2 f_{2}}\right)^{2}} \sqrt{\sum_{i=1}^{M}\left(A_{i}^{f_{1}+2 f_{2}}-\bar{A}_{1} f_{1}+2 f_{2}\right)^{2}}},
$$

where $\bar{A}_{i}{ }^{f 1-2 f 2}$ and $\bar{A}_{i}{ }^{f 1+2 f 2}$ are the mean values of the amplitude of the nonlinear components at $f_{1}-2 f_{2}$ and $f_{1}+2 f_{2}$, and $M$ is the level of uniform design tests, which is set to 15 . The correlation coefficient $P$ is a deflection major-type function meaning that the larger the value of $P$ is, the better is the consistency of the two mixing components.

To show the effect of two coefficients on evaluating the performance of magnetic frequency mixing testing, the two coefficients were calculated from the experimental signals. Table 5 lists some typical results, which were obtained from experiments conducted on the nine samples at different levels. The variational 
regularity of the two coefficients with the excitation parameters were different, and the corresponding optimal excitation parameters were different. More specifically, the excitation parameter of the 6th level is optimal as determined by the variation coefficient $D$, whereas the excitation parameter of the 11th level is optimal as determined by the correlation coefficient $P$.

Furthermore, Figure 6 shows some typical results to explain the dependence of magnetic frequency mixing testing on the preferred values of the excitation parameters. Figures $6 a, c$ and e present spectra of the detection signals obtained from sample 1 at three typical conditions; Figures $6 \mathrm{~b}, \mathrm{~d}$ and $\mathrm{f}$ show the amplitudes of the lower-order nonlinear components obtained from different samples under the three typical conditions.

Obviously, the excitation parameters preference has a great influence on the performance of magnetic frequency mixing testing. For excitation conditions at the 6th level, the amplitudes of the nonlinear components are the largest, and are one order greater in magnitude than those of the other two cases. Nevertheless, the difference in amplitude of the two lower-order nonlinear components is also larger; at the 11th level, the two nonlinear components are consistent in amplitude, but their values are relatively lower, whereas at the 5th level, the amplitudes of the two nonlinear components are relative small and have weak consistency, meaning that magnetic frequency mixing is weak. Overall the two coefficients only characterizes the magnetic frequency mixing effect in two different aspects.

\subsubsection{Fuzzy Model for Evaluating of Magnetic Frequency Mixing Effect}

Based on the above two coefficients, a comprehensive model was established to evaluate the effect of magnetic frequency mixing synthetically.

Given the relationship of the two coefficients in magnetic frequency mixing, the ridge function and the Cauchy function were selected as the subordination function of the variation coefficient $D$ and correlation coefficient $P$, respectively. Specifically, the step-down ridge function $\mu_{\mathrm{D}}^{\mathrm{R}}\left(x_{\mathrm{D}, j}\right)$ is given as
$\mu_{\mathrm{D}}^{\mathrm{R}}\left(x_{\mathrm{D}, j}\right)= \begin{cases}1, & x_{D, j} \leq 0.001, \\ \frac{1}{2}-\frac{1}{2} \sin \left(\frac{\pi}{0.499}\left(x_{\mathrm{D}, j}-\frac{0.501}{2}\right)\right), & 0.001<x_{\mathrm{D}, j} \leq 0.5, \\ 0, & x_{\mathrm{D}, j}>0.5,\end{cases}$

where $x_{\mathrm{D}, j}$ is the value of the variation coefficient obtained from the $j$ th level of uniform experimental design $(1 \leq j \leq 15)$. When $x_{\mathrm{D}, j} \leq 0.001$, the membership degree is 1 , which means that the value is completely accepted; When $0.001<x_{\mathrm{D}, j} \leq 0.5$, the membership degree decreases with the value of the ridge function until $\mu_{\mathrm{D}}^{\mathrm{R}}\left(x_{\mathrm{D}, j}\right)$ is zero $\left(x_{\mathrm{D}, j}>0.5\right)$, which means the value is not accepted.

Similarly, the step-up Cauchy function $\mu_{\mathrm{P}}^{C}\left(x_{\mathrm{P}, j}\right)$ is used to describe the membership degree distribution of the correlation coefficient $P$, its explicit expression being

$$
\mu_{\mathrm{P}}^{C}\left(x_{\mathrm{P}, j}\right)= \begin{cases}\frac{1}{1+1.257\left(0.999-x_{\mathrm{P}, j}\right)^{0.356}}, & x_{\mathrm{P}, j}<0.999, \\ 1 & x_{\mathrm{P}, j} \geq 0.999,\end{cases}
$$

where $x_{\mathrm{P}, j}$ is the value of the correlation coefficient obtained from the $j$ th level of the UED experiment. When $x_{\mathrm{P}, j}<0.999$, the membership degree increases with the Cauchy function until it is completely accepted. If $x_{\mathrm{P}, j} \geq 0.999$, the membership degree is 1 signifying that the value is completely accepted. Table 6 lists the membership degree of the two coefficients at different levels; Figure 7 plots their membership degree and fitted curves. Note that both $D$ and $P$ values are concentrated mainly in a range with a high level of satisfaction. Therefore, the weight of these two coefficients can be expressed by the average membership degree in Table 6.

With a consideration of the weights $w_{i}$ of the two coefficients, a fuzzy model is constructed

$$
\begin{aligned}
f(x)= & \sum_{i=1}^{n} w_{i} \mu_{A i}\left(x_{i, j}\right)=w_{1} \mu_{\mathrm{D}}^{\mathrm{R}}\left(x_{D, j}\right)+w_{2} \mu_{\mathrm{P}}^{\mathrm{C}}\left(x_{\mathrm{P}, j}\right) \\
= & 0.58\left(\frac{1}{2}-\frac{1}{2} \sin \left(\frac{\pi}{0.499}\left(x_{\mathrm{D}, j}-\frac{0.501}{2}\right)\right)\right) \\
& +0.42\left(\frac{1}{1+1.257\left(0.999-x_{\mathrm{P}, j}\right)^{0.356}}\right) .
\end{aligned}
$$

Table 5 Variation coefficient and correlation coefficient obtained from all samples at different levels

\begin{tabular}{lllllllr}
\hline Level & $\mathbf{1}$ & $\mathbf{2}$ & $\mathbf{3}$ & $\mathbf{4}$ & $\mathbf{5}$ & $\mathbf{6}$ & $\mathbf{7}$ \\
\hline$x_{D}$ & 0.3070 & 0.3181 & 0.1441 & 0.0296 & 0.1629 & 0.0132 \\
$x_{P}$ & -0.3078 & 0.7330 & -0.0677 & 0.9078 & -0.8602 & -0.0710 & 0.0757 \\
\hline Level & $\mathbf{9}$ & $\mathbf{1 0}$ & $\mathbf{1 1}$ & $\mathbf{1 2}$ & $\mathbf{1 3}$ & $\mathbf{1 4}$ \\
\hline$x_{D}$ & 0.0256 & 0.0356 & 0.0825 & 0.0692 & 0.0679 & 0.2305 \\
$x_{P}$ & -0.1638 & 0.9628 & 0.9979 & 0.8282 & 0.9613 & 0.0904 \\
\hline
\end{tabular}

Optimal values are in italics 


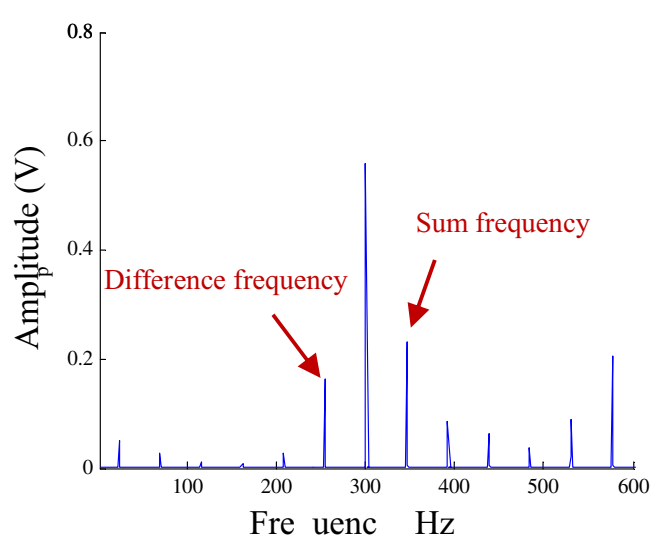

a Spectrum obtained from sample 1, and excited at 6 th level

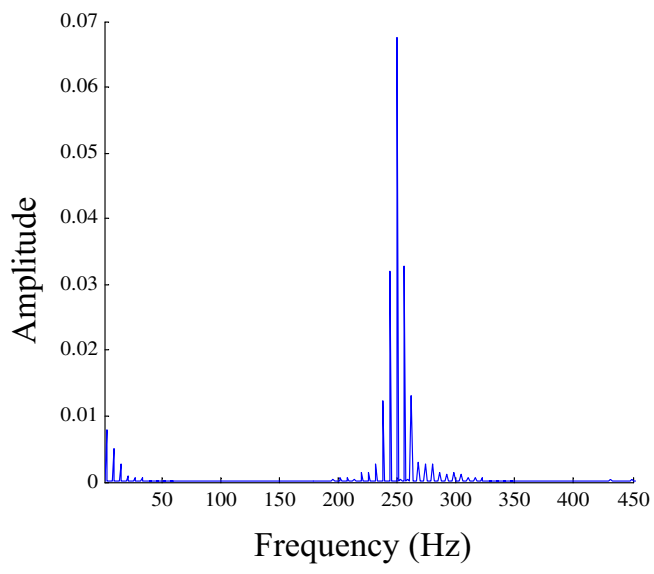

c Spectrum obtained from sample 1, and excited at 11th level

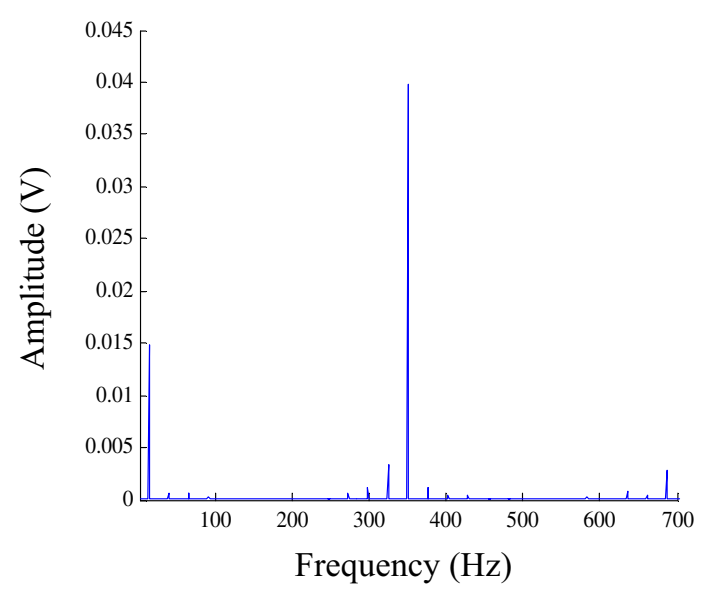

e Spectrum obtained from sample 1, and excited at 5th level

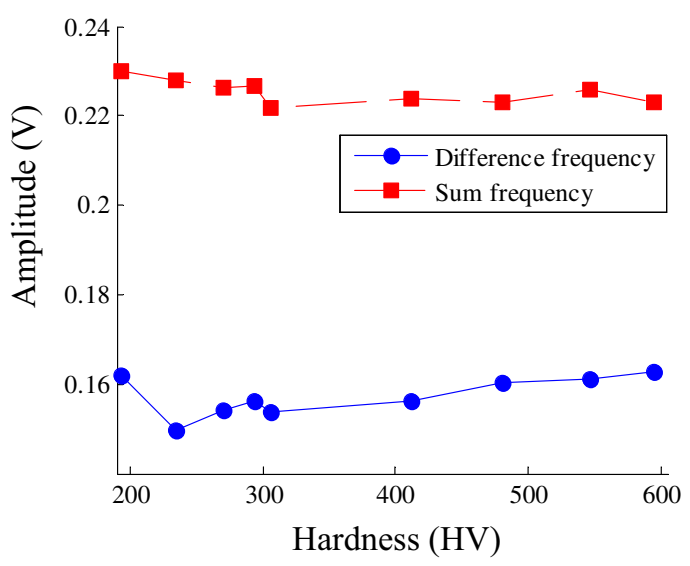

b Amplitude of frequency mixing components when the excitation condition is the 6th level

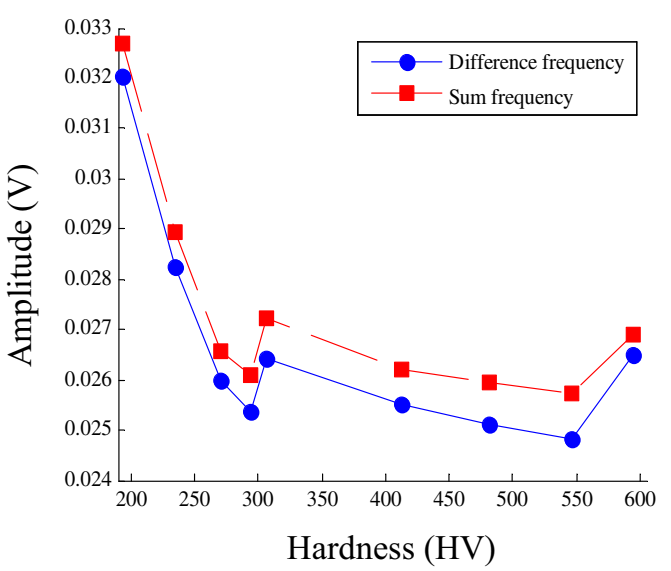

d Amplitude of frequency mixing components when the excitation condition is the 11th level

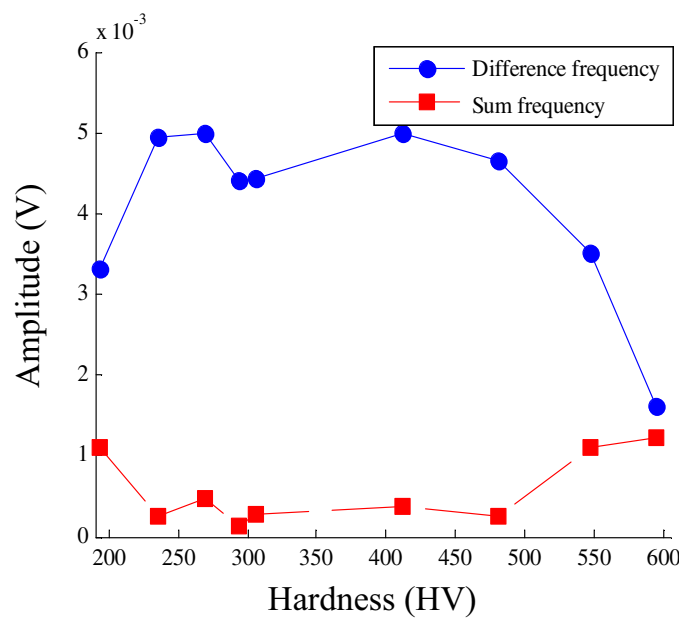

f Amplitude of frequency mixing components when the excitation condition is the 5th level

Figure 6 Dependence of magnetic frequency mixing effect on excitation parameters 
Table 6 Membership function values of two evaluation indexes at different uniform design levels

\begin{tabular}{lllllllll}
\hline Level & $\mathbf{1}$ & $\mathbf{2}$ & $\mathbf{3}$ & $\mathbf{4}$ & $\mathbf{5}$ & $\mathbf{6}$ & $\mathbf{7}$ \\
\hline$\mu_{\mathrm{D}}^{\mathrm{R}}$ & 0.3259 & 0.2936 & 0.8104 & 0.9919 & 0.7620 & 0.9985 & 0.9857 \\
$\mu_{\mathrm{p}}^{C}$ & 0.4197 & 0.5604 & 0.4374 & 0.6511 & 0.3895 & 0.4371 & 0.4915 \\
\hline Level & $\mathbf{9}$ & $\mathbf{1 0}$ & $\mathbf{1 1}$ & $\mathbf{1 2}$ & $\mathbf{1 3}$ & $\mathbf{1 4}$ & $\mathbf{1 5}$ & 0.9457 \\
\hline$\mu_{\mathrm{D}}^{\mathrm{R}}$ & 0.9940 & 0.9882 & 0.9356 & 0.9546 & 0.9563 & 0.5628 & 0.9229 \\
$\mu_{\mathrm{p}}^{C}$ & 0.4299 & 0.7217 & 0.8999 & 0.5988 & 0.7188 & 0.5854 & 0.7911 \\
\hline
\end{tabular}

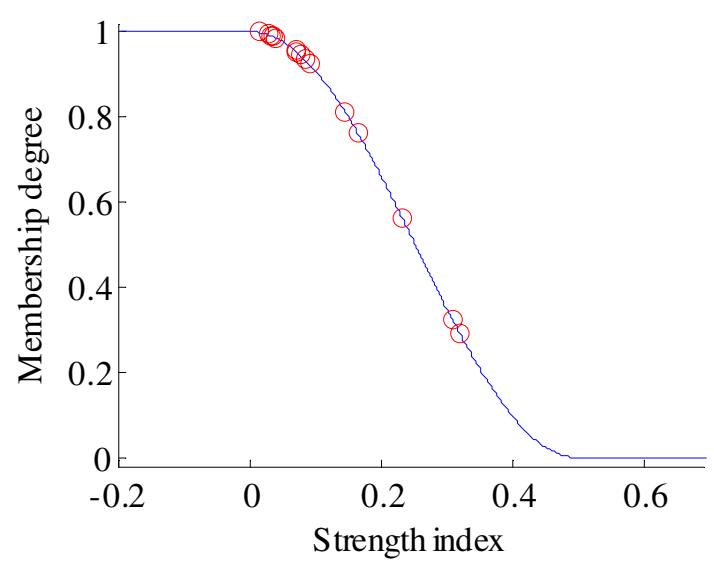

a Ridge function for variation coefficient

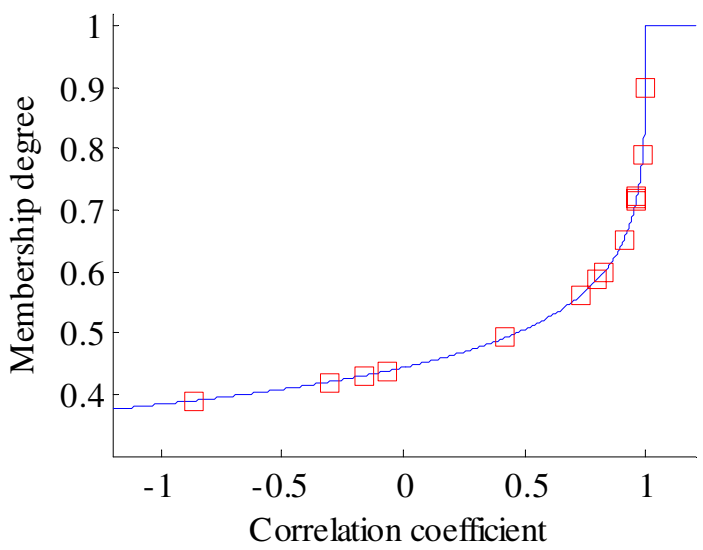

b Cauchy function for correlation coefficient

Figure 7 Curves of the two subordination functions

The frequency-mixing effect can now be characterized more accurately using this fuzzy evaluation function.

\subsection{Regression Analysis}

The fuzzy evaluation function in Eq. (10) is an implicit expression of the excitation parameters, and therefore, it cannot be used directly in the optimization of the
Table 7 Correlation indices of the stepwise regression analysis

\begin{tabular}{llll}
\hline Correlation index & $\boldsymbol{R}$ & $\boldsymbol{R}^{\mathbf{2}}$ & $\boldsymbol{F}$ \\
\hline Results & 0.995 & 0.990 & 55.139
\end{tabular}

excitation parameters. In this section, a polynomial is chosen as the regression function to model explicitly the correlation between the excitation parameters and frequency-mixing effect. The above fuzzy evaluation function $f(x)$ may be expressed as a general quadratic

$$
f(x)=\beta_{0}+\sum_{i=1}^{m} \beta_{i} x_{i}+\sum_{i=1}^{m} \beta_{i i} x_{i}^{2}+\sum_{i<j} \beta_{i j} x_{i} x_{j},
$$

where $x_{i}$ denotes an independent variable, $\beta_{0}, \beta_{i}, \beta_{i i}$ and $\beta_{i j}$ are the regression coefficients of the model, and $m$ is the number of independent variables, here set to 4 . In this study, $x_{1}$ and $x_{2}\left(x_{3}\right.$ and $\left.x_{4}\right)$ are the frequency and amplitude of the low- (high-) frequency magnetic field, respectively. Then, the influence of the excitation parameters on frequency mixing is evaluated in a stepwise regression analysis. Table 7 lists the values of the correlation indices from this analysis; $R$ is the adjustment correlation coefficient, $R^{2}$ the coefficient of determination and $F$ the overall F-statistic. If the values of $R$ and $R^{2}$ are close to 1 and the value of $F$ is more than 0.05 , the regression analysis is considered to have fully converged.

The explicit expression of the fuzzy evaluation function in terms of the excitation parameters and magnetic frequency mixing is

$$
\begin{aligned}
f(x)= & 0.177-0.008 x_{1}+0.11 x_{3}+0.227 x_{4} \\
& -0.059 x_{4}^{2}-0.002 x_{1} x_{3}+0.004 x_{1} x_{4} \\
& +3.461^{-5} x_{2} x_{3}-0.015 x_{3} x_{4} .
\end{aligned}
$$

Given the reasonable ranges of each excitation parameter (Table 3), the excitation parameters were then optimized using the genetic algorithm. Table 8 lists these optimal results. 


\begin{tabular}{|c|c|c|c|c|}
\hline $\begin{array}{l}\text { Fuzzy } \\
\text { evaluation } \\
\text { index }\end{array}$ & $\begin{array}{l}\text { Low } \\
\text { amplitude } \\
\text { (V) }\end{array}$ & $\begin{array}{l}\text { High } \\
\text { amplitude } \\
\text { (V) }\end{array}$ & $\begin{array}{l}\text { Low } \\
\text { frequency } \\
(\mathrm{Hz})\end{array}$ & $\begin{array}{l}\text { High } \\
\text { frequency } \\
(\mathrm{Hz})\end{array}$ \\
\hline 1.2708 & 5 & 1 & 1 & 200 \\
\hline
\end{tabular}

Table 9 Excitation parameters of contrastive experiment

\begin{tabular}{llllll}
\hline $\begin{array}{l}\text { No. } \\
\begin{array}{l}\text { Fuzzy } \\
\text { evaluation } \\
\text { value }\end{array}\end{array}$ & $\begin{array}{l}\text { Low } \\
\text { amplitude } \\
\text { (V) }\end{array}$ & $\begin{array}{l}\text { High } \\
\text { amplitude } \\
\text { (V) }\end{array}$ & $\begin{array}{l}\text { Low } \\
\text { frequency } \\
\text { (Hz) }\end{array}$ & $\begin{array}{l}\text { High } \\
\text { frequency } \\
\text { (Hz) }\end{array}$ \\
\hline 1 & 1.2708 & 5 & 1 & 1 & 200 \\
2 & 0.9206 & 5.5 & 0.5 & 3 & 250 \\
3 & 0.3653 & 2 & 2.7 & 17 & 700 \\
\hline
\end{tabular}

\section{Experimental Validation}

To validate the scheme with the preferred optimal excitation parameter settings, comparison experiments under different conditions were conducted using the surface hardness measurements of specimens (Table 2). Table 9 gives the specific parameters of the experiments. Note that the first group has the optimal parameter settings obtained from the regression analysis; the other two groups were arbitrarily chosen from the UED table (Table 4).

Similarly, Figure 8 gives some typical results to show the dependence of magnetic frequency mixing on the excitation parameter preferences. Figures $8 \mathrm{a}, \mathrm{c}$ and e present spectra of the detection signals obtained from one sample at three typical conditions; Figures 8b, d and $f$ present the amplitude of lower-order nonlinear components obtained from different samples at three typical conditions.

The results are similar to Figure 6. The excitation parameter preferences have clearly a great influence on the performance of magnetic frequency mixing detection. Specifically, when the hardness measurement was conducted using optimal excitation parameters, the amplitudes of the lower-order nonlinear components are the largest, and they are one order greater in magnitude than those of the other two instances; moreover, the two lower-order nonlinear components show greater consistency in amplitude than those of the other two instances (Figure 9). The implication is that magnetic frequency mixing is strongest under this condition. In addition, Figures $8 \mathrm{~b}, \mathrm{~d}$ and $\mathrm{f}$ show the relationship between mixing amplitudes and surface hardness. The regression coefficients were also calculated. The best relation is obtained from the optimal excitation parameters. The high regression coefficients indicate the advantages of the optimized excitation parameters for hardness detection. Besides, it can be seen from the results that the error of detection results is different under different excitation conditions. Based on the first and second group of excitation parameters, the error of detection results is generally small. For the third one, the detection error of individual results is larger. Therefore, the magnetic frequency mixing testing under the optimal excitation parameter has greater sensitivity, better linearity and robustness, and is more suitable for the hardness measurement of ferromagnetic materials.

\section{Conclusions}

The UED method was used to analyze the influence of excitation parameters on the performance of magnetic frequency mixing detection. These excitation parameters were optimized using a regression analysis and a genetic algorithm. The conclusions of the study can be summarized as follows:

(1) The excitation parameters preference has a great influence on the performance of magnetic frequency mixing detection;

(2) Frequency-mixing effect can be characterized more accurately using a multi-index fuzzy evaluation function;

(3) Optimal excitation parameter settings for magnetic frequency mixing detection were obtained (see Table 8);

(4) The magnetic frequency mixing testing under the optimal excitation parameter settings have greater sensitivity and better linearity, and hence more suitability in hardness measurements of ferromagnetic materials. 


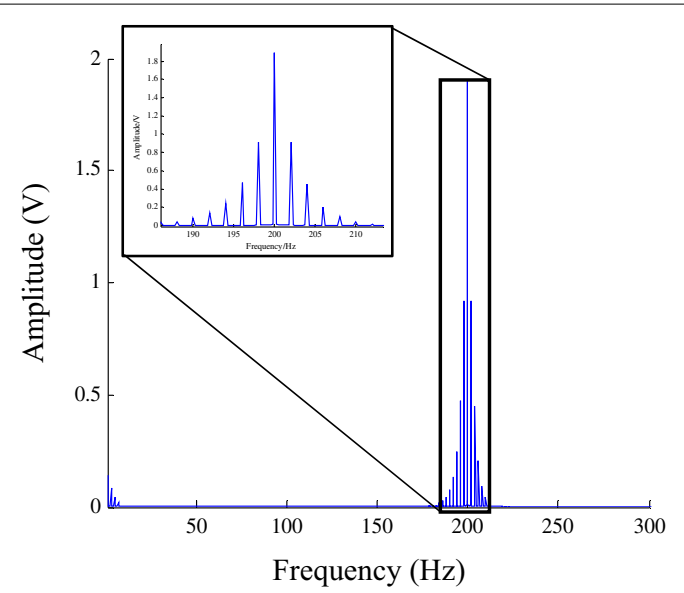

a Spectrum obtained from sample 1, and excited at the 1st level

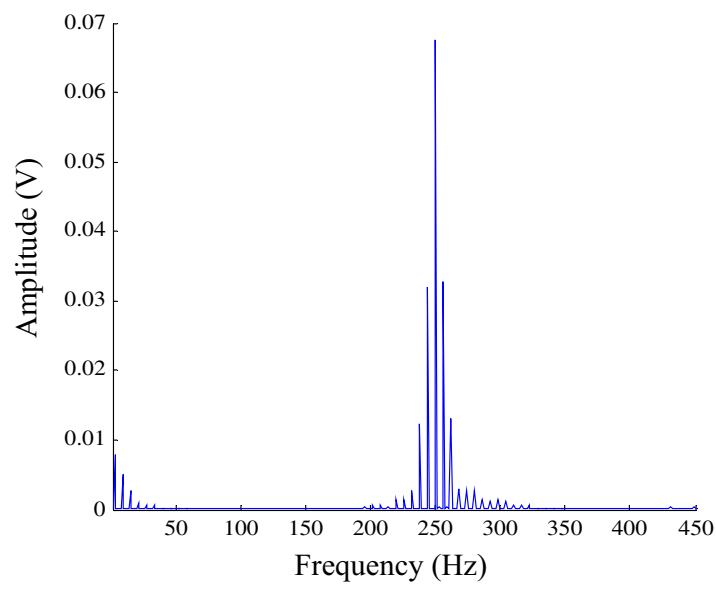

c Spectrum obtained from sample 1, and excited at the 2 nd level

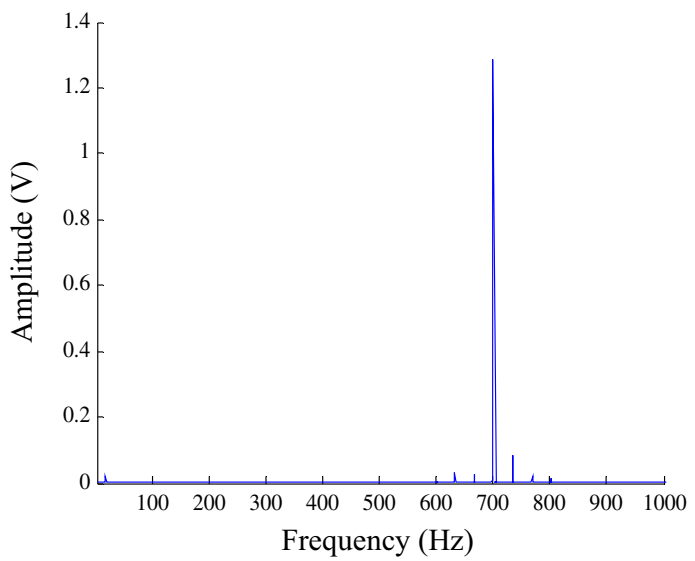

e Spectrum obtained from sample 1, and excited at the 3 rd level

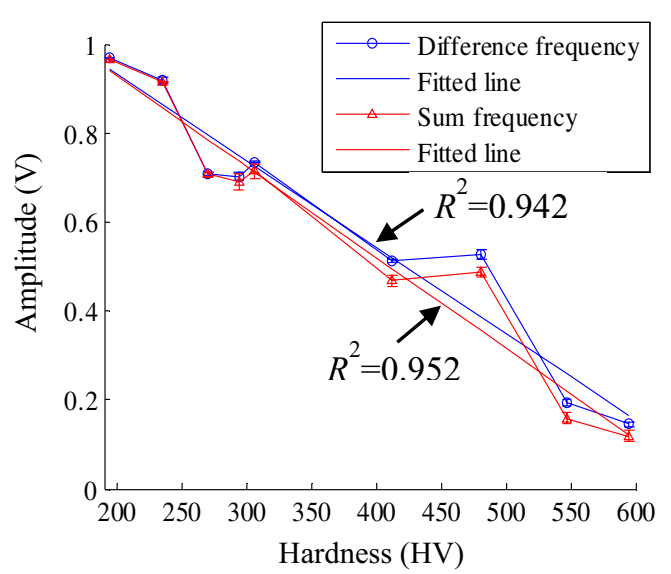

b Amplitude of frequency mixing components when the excitation condition is the 1st level

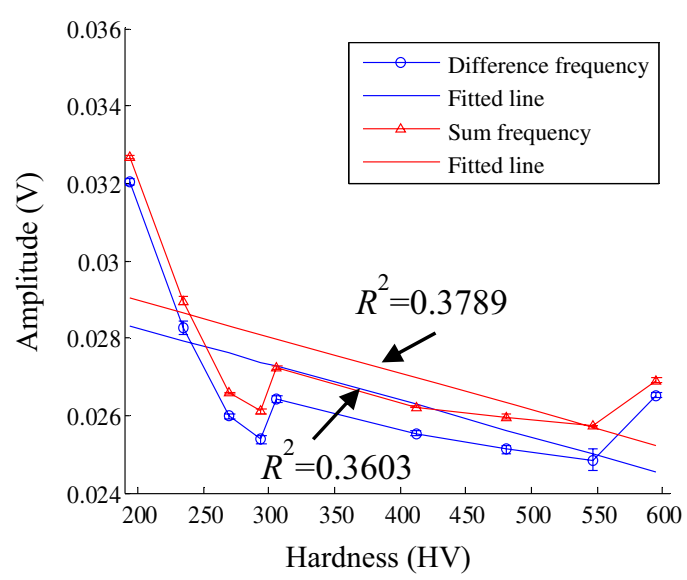

d Amplitude of frequency mixing components when the excitation condition is the 2 nd level

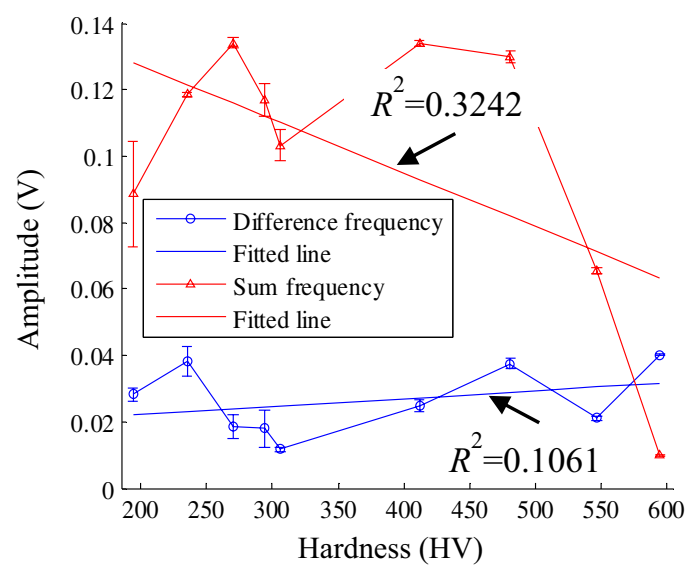

f Amplitude of frequency mixing components when the excitation condition is the 3rd level

Figure 8 Dependence of magnetic frequency mixing effect on excitation parameters 


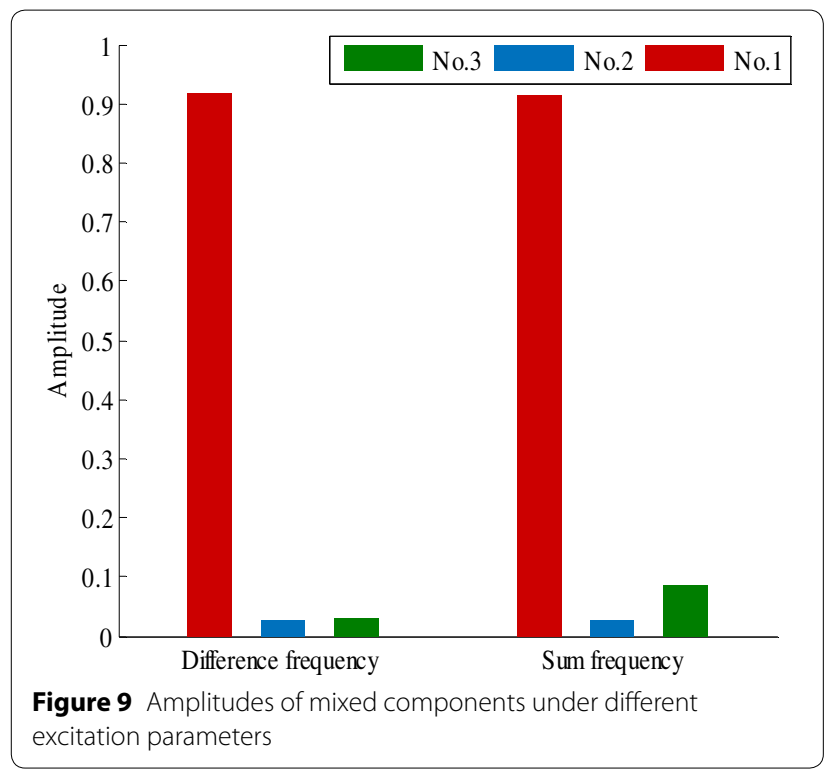

\section{Acknowledgements}

We thank Richard Haase, PhD, from Liwen Bianji, Edanz Group China (www. liwenbianji.cn/ac), for editing the English text of a draft of this manuscript.

\section{Authors' Contributions}

$Y C$ and JJ conceived and designed the study. YC and XL performed the experiments. $G L$ and $C H$ provided the experimental instruments. $Y C$ and JJ wrote the paper. $\mathrm{XL}, \mathrm{GL}, \mathrm{CH}$ and $\mathrm{BW}$ reviewed and edited the manuscript. All authors read and approved the final manuscript.

\section{Authors' Information}

Yu Chang received her bachelor degree in 2013 from Sichuan University. She is currently a PhD candidate at Beijing University of Technology, China. Her main research interests include non-destructive testing and automatic control.

Jingpin Jiao was born in 1973 in Hebei (China). She received her BS degrees in Mechanical Engineering in 1998 from Yanshan University, and PhD degree in 2005 from Beijing University of Technology, China. She is currently full professor at College of Mechanical Engineering and Applied Electronics Technology, Beijing University of Technology, China. Her main research interests include nondestructive testing of new technology, experimental solid mechanics, modern measurement and control technology, and new sensor technology.

Xiucheng Liu received his BS and PhD degree in 2007 and 2013 from Beijing University of Aeronautics and Astronautics and Beijing University of Technology respectively. He is currently a professor at Beijing University of Technology. His main research interests include micro-magnetic nondestructive measurement method for mechanical properties, sensor technology, and nondestructive testing technology.

Guanhai Li received his BS, MS and PhD degree in 1994, 1998 and 2002 from South China University of Technology. He is currently a researcher in China Special Equipment Inspection and Research Institute. His main research interests include non-destructive testing techniques and its application in special equipment inspection.

Cunfu He received his BS, MS and PhD degree in1985, 1990 and 1996 from Taiyuan University of Technology, Huazhong University of Science and Technology and Tsinghua University, respectively. He is currently a professor at Beijing University of Technology. His main research interests include mechanical testing theory method and technology, ultrasonic nondestructive testing technology, and sensor technology.

Bin Wu received his BS, MS and PhD degree in 1984, 1990 and 1996 from Tianjin University, Beijing University of Aeronautics and Astronautics and Taiyuan University of Technology, respectively. He is currently a professor at Beijing University of Technology. His main research interests include experimental solid mechanics, modern measurement and control technology, nondestructive testing of new technology, and new sensor technology.

\section{Funding}

Supported by National Key Research and Development Program of China (Grant No. 2017YFF0209703) and National Natural Science Foundation of China (Grant Nos. 11972053, 11527801).

\section{Competing Interests}

The authors declare no competing financial interests.

\section{Author Details}

${ }^{1}$ College of Mechanical Engineering and Applied Electronics Technology, Beijing University of Technology, Beijing 100124, China. ${ }^{2}$ China Special Equipment Inspection and Research Institute, Beijing 100013, China.

Received: 21 March 2019 Revised: 30 December 2019 Accepted: 9 January 2020

Published online: 10 February 2020

\section{References}

[1] I Teliban, C Thede, S Chemnitz, et al. Magnetic moment investigation by frequency mixing techniques. Review of Scientific Instruments, 2009, 80(11): 631-635.

[2] H J Krause, N Wolters, Y Zhang, et al. Magnetic particle detection by frequency mixing for immunoassay applications. Journal of Magnetism \& Magnetic Materials, 2007, 311(1): 436-444.

[3] T L Paoli, J F Svacek. Derivative measurement by frequency mixing. Review of Scientific Instruments, 1976, 47(9): 1016-1019.

[4] LTu, Y Feng, T Klein, et al. Measurement of Brownian relaxation of magnetic nanoparticle by a multi-tone mixing-frequency method. IEEE Transactions on Magnetics, 2012, 48(11): 3513-3516.

[5] Yu C, Jingpin J, Xiucheng $L$, et al. Measurement of the hardness of medium carbon steel using the magnetic mixing-frequency technique. IEEE Transactions on Magnetics, 2018, 54(12): 1-7.

[6] BW Ficko, P Giacometti, S G Diamond. Nonlinear susceptibility magnitude imaging of magnetic nanoparticles. Journal of Magnetism \& Magnetic Materials, 2015, 378: 267-277.

[7] L Tu, K Wu, T Klein, et al. Magnetic nanoparticles colourization by a mixing-frequency method. Journal of Physics D Applied Physics, 2014, 47(15): 494-500.

[8] L Tu, Y Jing, Y Li, et al. Real-time measurement of Brownian relaxation of magnetic nanoparticles by a mixing-frequency method. Applied Physics Letters, 2011, 98(21): 973-975.

[9] DC Jiles, Y Melikhov. Modelling of nonlinear behaviour and hysteresis in magnetic materials. In: Handbook of Magnetism and Advanced Magnetic Materials, John Wiley \& Sons, Ltd, 2007.

[10] S Xie, Z Chen, T Takagi, et al. Development of a very fast simulator for pulsed eddy current testing signals of local wall thinning. NDT \& E International, 2012, 51(10): 45-50.

[11] H Xu, Y Pei, D Fang, et al. Nonlinear harmonic distortion effect in magnetoelectric laminate composites. Applied Physics Letters, 2014, 105(1): 418-421.

[12] M Vashista, V Moorthy. Influence of applied magnetic field strength and frequency response of pick-up coil on the magnetic barkhausen noise profile. Journal of Magnetism \& Magnetic Materials, 2013, 345(10): 208-214.

[13] A F Chávez-González, J A Pérez-Benítez, J H Espina-Hernández, et al. Influence of frequency of the excitation magnetic field and material's electric conductivity on domain wall dynamics in ferromagnetic materials. Journal of Magnetism \& Magnetic Materials, 2016, 401: 287-295.

[14] S Xie, Z Chen, T Takagi, et al. Quantitative non-destructive evaluation of wall thinning defect in double-layer pipe of nuclear power plants using pulsed ECT method. NDT \& E International, 2015, 75(10): 87-95.

[15] D A Burdin, D V Chashin, N A Ekonomov, et al. Nonlinear magnetoelectric effects in ferromagnetic-piezoelectric composites. Journal of Magnetism \& Magnetic Materials, 2014, 358-359(5): 98-104.

[16] A Rabehi, B Garlan, S Achtsnicht, et al. Magnetic detection structure for lab-on-chip applications based on the frequency mixing technique. Sensors, 2018, 18(6): 1747-1760. 
[17] Bo Chen, Dianrong Gao, Chao Yang, et al. Multi-objective intelligent collaborative optimization of structure parameters for high-power remote sprayer. Journal of Mechanical Engineering, 2017, 53(6): 166-175. (in Chinese)

[18] N Li, X Yang, Y Gong, et al. Enhancing electrical capacitance tomographic sensor design using fuzzy theory based quantifiers. Measurement Science \& Technology, 2014, 25(12): 125-134.

[19] N Li, M Cao, C He, et al. Multi-parametric indicator design for ect sensor optimization used in oil transmission. IEEE Sensors Journal, 2017, 17(7): 2074-2088.

[20] KT Fang, C Ma, P Winker, et al. Uniform design: Theory and application. Technometrics, 2000, 42(3): 237-248.

[21] Dengwan Li, Hongtao Chen, Jinchun Feng, et al. Precision cutting parameters optimization based on uniform design method. Journal of Mechanical Engineering, 2015, 51(3): 206-212. (in Chinese)

[22] L Deng, B Feng, Y Zhang. An optimized method for multi-objective and multi-factor designing of a ceramic slurry: combining orthogonal experimental design with artificial neural networks. Ceramics International, 2018, 44(6): 15918-15923.

[23] B Wu, Y J Wang, X C Liu, et al. A novel TMR-based MFL sensor for steel wire rope inspection using the orthogonal test method. Smart Material Structures, 2015, 24(7): 7-18.
[24] H Ping, G Xu, S Wu. System optimization of cyclohexane dehydrogenation under multiphase reaction conditions using the uniform design method. International Journal of Hydrogen Energy, 2015, 40(46): 15923-15932.

[25] G Song, G Xu, Y Quan, et al. Uniform design for the optimization of Al2O3 nanofilms produced by electrophoretic deposition. Surface and Coatings Technology, 2016, 286(12): 268-278.

[26] J Yang, L Li, L Yang, et al. Uniform design for the parameters optimization of pin-fins channel heat sink. Applied Thermal Engineering, 2017, 120(3): 289-297.

[27] H Wang, L Zhang, G Li, et al. Application of uniform design experimental method in waste cooking oil (WCO) co-hydroprocessing parameter optimization and reaction route investigation. Fuel, 2017, 210(9): 390-397.

[28] J TTsai, PY Yang, J H Chou. Data-driven approach to using uniform experimental design to optimize system compensation parameters for an auto-alignment machine. IEEE Access, 2018, 6: 40365-40378.

[29] SW Huang, J H Chou, J T Tsai. Uniform design and regression analysis methods for optimal operational parameter design of high-pressure waterjet machine. International Journal of Automation and Smart Technology, 2018, 8(2): 85-88

\section{Submit your manuscript to a SpringerOpen ${ }^{\circ}$ journal and benefit from:}

- Convenient online submission

- Rigorous peer review

- Open access: articles freely available online

- High visibility within the field

- Retaining the copyright to your article

Submit your next manuscript at $\boldsymbol{\nabla}$ springeropen.com 